\title{
Requiem pour une morte : Aftermath (Cerdà 1994)
}

Ou l'art paradoxal de réhumaniser le cadavre

Requiem for a Dead Lady: Aftermath (Cerdà 1994) or the Paradoxical Art of

Rehumanising the Corpse

\section{Marika Moisseeff}

\section{(2) OpenEdition \\ Journals}

Édition électronique

URL : https://journals.openedition.org/tc/6991

DOI : $10.4000 /$ tc. 6991

ISBN : 1952-420X

ISSN : 1952-420X

Éditeur

Éditions de l'EHESS

\section{Édition imprimée}

Date de publication : 19 juin 2013

Pagination : 160-179

ISBN : 978-2-7351-1637-9

ISSN : 0248-6016

Référence électronique

Marika Moisseeff, «Requiem pour une morte : Aftermath (Cerdà 1994) », Techniques \& Culture [En ligne], 60 | 2013, mis en ligne le 19 juin 2016, consulté le 29 septembre 2022. URL : http:// journals.openedition.org/tc/6991; DOI : https://doi.org/10.4000/tc.6991 


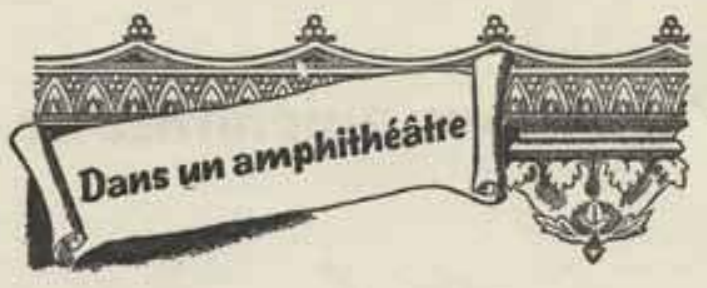

$$
-1-
$$

Dans un amphitbetative (bis) Macralues maccabes, meccibes Troin, twoin )

$$
-2-
$$

Ce maccable $m^{\prime}$ dinait (biv)

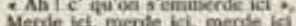
Troin, tsotn ?

$$
-3-
$$

Alos on Tenpueula $(\mathrm{Big})$ En puis on r dereg Dipesa, depeca, itepeca

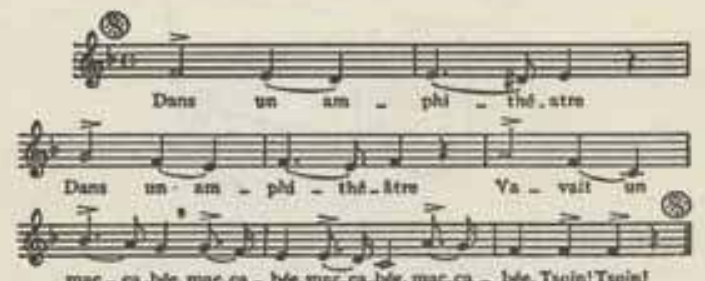

$$
-4-
$$

On le disstquera (bir)

Avec un speculum

Spoculum, speculum, speculam Troia, tsaia

$$
-5-
$$

On le coenervera (bis)

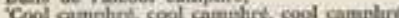
Toin, twotat

$$
-6-
$$

Muis on le bouffura (Mis)

Det on te destenira.

desuralra Tsoin, tein

$-7-$

Enfin on t'enterr'ra (bis)

- Dies ince, dirs ifla * Troin, trotin ?

$$
-8-
$$

Sar a tambe on mettra (bis)

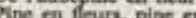

feun, pine en feurs, plee en fleurs Troin, twotn I 


\section{REQUIEM POUR UNE MORTE : AFTERMATH (CERDÄ 1994)}

\section{Ou l'art paradoxal de réhumaniser le cadavre}

La mort, loin d'être devenue un sujet tabou dans les sociétés occidentales contemporaines, comme il est d'usage de l'affirmer depuis quelques décennies ${ }^{1}$, est au contraire rendue omniprésente dans les médias : elle fait la une des journaux papier ou télévisées, constitue la trame de nombreuses fictions littéraires et cinématographiques, de séries télévisées américaines très prisées et de témoignages autobiographiques, tandis que tout un chacun est sommé, de manière itérative et insistante, de faire un travail de deuil et/ ou de mémoire vis-à-vis de ses défunts. En revanche, il est vrai que la réalité concrète du cadavre tend, elle, à être occultée, phénomène qui ne peut échapper à l'ethnologue travaillant dans des sociétés autres où sa présence préside à la structuration des rites funéraires et à la gestion des réactions émotionnelles des proches. Dans l'Occident d'aujourd'hui, le cadavre humain et son traitement tendent, en effet, à être l'exclusive des institutions médico-chirurgicales et médico-légales et l'analyse des procédures concrètes et réelles qui s'y déroulent est bien moins médiatisée ; on peut cependant relever qu'elles sont elles aussi le sujet de fictions (séries et romans policiers américains) de plus en plus nombreuses et ce depuis relativement récemment. Les réactions émotionnelles des endeuillés tendent, pour leur part, à être gérées à distance de cette présence lourde et abjecte de la décomposition de l'être aimé, notamment au travers des conseils prodigués par les psys en tous genres. Cet article propose de voir dans la multiplication des films d'horreur une façon de reconnecter l'abjection du corps mort avec les réactions fortes qu'elle suscite lorsque les personnes y sont directement confrontées. Or, s'il revient aux institutions médico-chirurgicales de faire le « sale boulot » à l'écart du monde profane pour rendre aux morts un aspect tolérable, il revient, par contre, aux films d'horreur de réintroduire 
Dans un amphithéâtre (Ter) Phithéâtre, phithéâtre, phithéâtre,

Tsouin, tsouin!

Y'avait un macchabée (Ter)

Macchabée (Ter)

Tsouin, tsouin

Qui sentait fort des pieds (Ter)

Fort des pieds (Ter)

Tsouin, tsouin

Ce macchabée disait (Ter)

Il disait (Ter)

Tsouin, tsouin

Ce macchabée gueulait (Ter)

Il gueulait (Ter)

Tsouin, tsouin

«Ah! c'qu'on s'emmerde ici (Ter)

Merde ici, (Ter)

Tsouin, tsouin

On va le disséquer (Ter)

Disséquer (Ter)

Tsouin, tsouin

Avec un spéculum (Ter)

Spéculum (Ter)

Tsouin, tsouin

On enf'ra du pâté (Ter)

Du pâté (Ter)

Tsouin, tsouin

Qui nous f'ra dégueuler (Ter)

Dégueuler (Ter)

Tsouin, tsouin

Chanson de salle de garde la dimension effroyable des corps morts en voie de putréfaction. C'est du moins l'hypothèse que tentera de soutenir cet article en montrant que si la gestion du cadavre renvoie, dans le monde réel, à des formes de transgression légitimées par l'appareil juridique, il faut ajouter de la transgression à la transgression légitime pour restituer, sur un plan fictionnel, son véritable aspect horrifique et son aptitude à nous affecter profondément. Pour étayer cette hypothèse, je vais me tourner vers le court-métrage Aftermath réalisé par Nacho Cerdà en 1994 qui occupe une place tout à fait singulière dans le cinéma d'horreur en raison de son caractère tout à la fois austère et particulièrement réaliste.

\section{La contingence de la mort}

Dans ce court-métrage de trente minutes, le générique d'ouverture qui dure quatre minutes fait partie intégrante du dispositif : à partir des différents plans s'insérant entre la présentation des noms et du statut ou rôle des personnes mentionnées, il permet de planter le décor. Or, dans ce film où ne sera prononcée aucune parole sinon celles à peine audibles du chœur du Requiem de Mozart lors de séquences précises, le décor avec les cadavres et les sons qui en font partie va devenir l'un des protagonistes essentiels. Il s'agit de dévoiler progressivement aux profanes que nous sommes ce qui, dans une société ayant pourtant fait vœu de transparence, nous demeure ordinairement obstinément caché de la gestion de nos corps une fois que les professionnels de la mort en disposent derrière les murs de la morgue. Cette initiation va, en effet, nous conduire, sinon à toucher et à sentir, du moins à voir et à entendre ce qui est généralement occulté du traitement habituel du cadavre dans les instituts médico-légaux. Nous serons ainsi à même de ressentir l'effroi dont on tend à nous préserver à toute force.

Plan noir. La porte d'une voiture que l'on ne voit pas claque. On entend le véhicule s'éloigner. Visualisation floue d'un cœur dont on entend par contre distinctement les battements. Sons de la voiture allant de plus en plus vite. Bruits de dérapage, grincements de freins, hurlements d'une femme, gémissements d'un animal. Apparition du nom du réalisateur en blanc sur fond noir, puis le blanc des lettres se transforme en rouge.

Plan d'une étendue de neige blanche et terne évoquant un jour froid d'hiver au petit matin sur laquelle apparaît une marre de sang rouge puis la masse étalée d'un gros chien dont les yeux grands ouverts sont immobiles, la bête étant à l'évidence morte, une partie de ses intérieurs étant répandue sur la neige. C'est alors que le titre du film surgit : Aftermath, ce qui renvoie à l'après-coup d'une catastrophe tel un holocauste. Ici, il s'agit des suites d'un accident de voiture mortel que nous allons suivre ; événement banal, malheureusement susceptible d'arriver à tout un chacun. Contraste donc entre la trivialité d'un événement du quotidien et l'irréversibilité de ses conséquences : c'est le thème même du film, celui de la transformation soudaine d'un sujet vivant en pur objet de chair inanimé. 


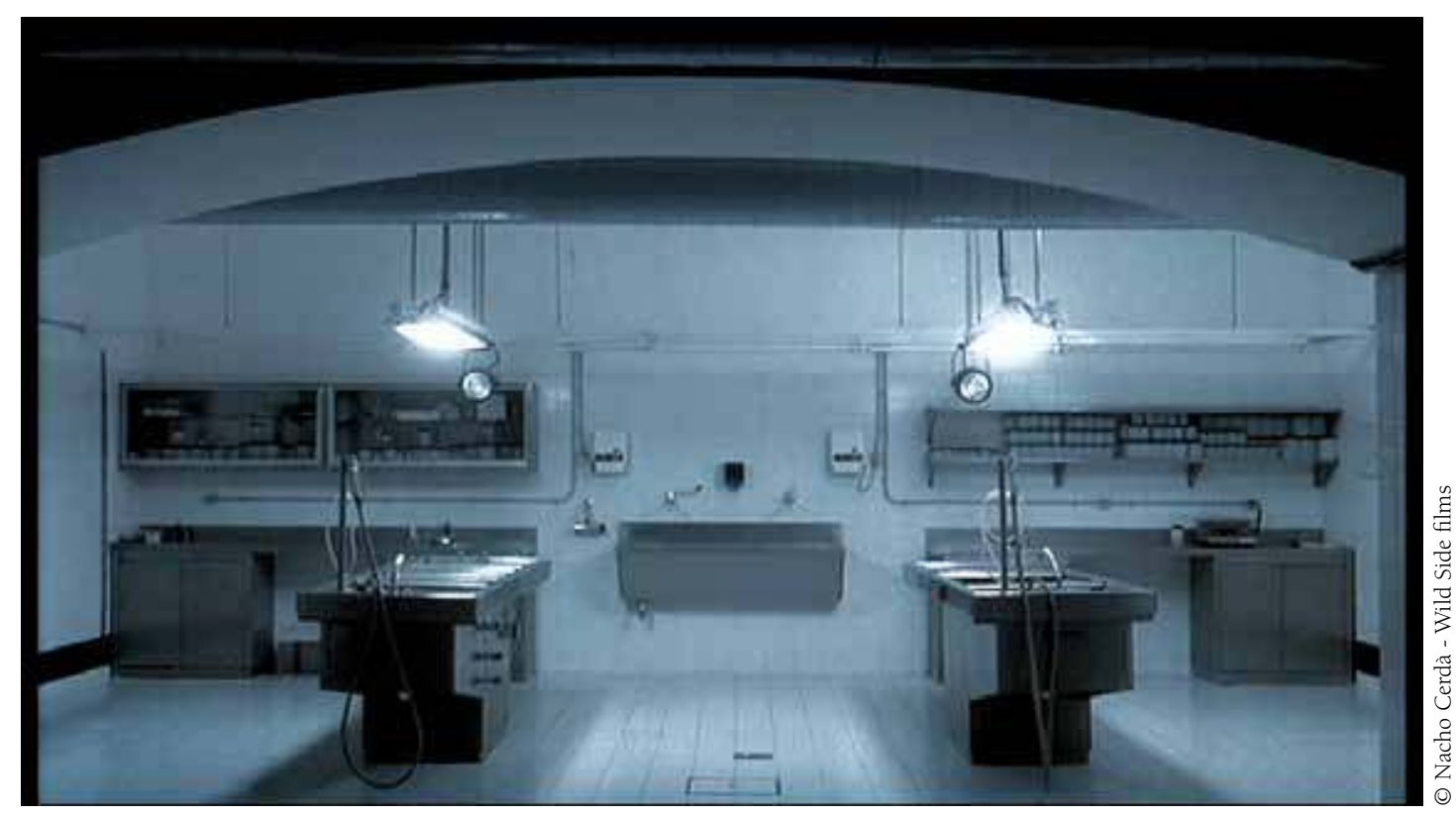

\section{La descente aux enfers}

On aperçoit la tête d'une jeune femme allongée sur un brancard juste au moment où on la lui recouvre du drap blanc qui couvre déjà le reste de son corps. On ne voit plus d'elle que ses orteils émergeant de ce linceul ; à son gros orteil est attachée une banale étiquette portant son nom: Marta Arnau. Cette défunte encore fumante va être notre Eurydice : en suivant sa trajectoire, nous allons, tel Orphée, traverser la frontière qui sépare les vivants et les morts en descendant peu à peu dans les tréfonds de l'hôpital ${ }^{2}$, à l'exemple de Dante parcourant les différents cercles de l'Enfer. Le véhicule de ce passage est un ascenseur franchissant un à un les soussols successifs. Chacun des paliers permet de nous introduire un peu plus avant dans le Saint des Saints où se gère l'irrémédiable, en nous en révélant chacun des éléments de sa structure avant que nous y découvrions la matière qui y est traitée.

Niveau moins un, nous apercevons les armoires frigorifiques métalliques verticales contenant les tiroirs où l'on entrepose les cadavres. Les lumières de deux scialytiques s'allument en même temps au-dessus de deux tables d'autopsie massives, métalliques, révélant une salle de morgue carrelée entièrement de blanc divisée en deux parties semblables : au fond un grand évier en inox à deux robinets, flanqué de part et d'autre d'une armoire métallique au-dessus de laquelle est disposée une étagère contenant des récipients en plastique vides. Ordre, propreté et symétrie presque parfaite.

Niveau moins deux. On distingue une petite croix en argent au bout d'une chaîne sur une surface métallique grise et froide. Le message est clair : la foi de la jeune femme ne l'a pas protégée. On entend un claquement de porte qui se referme (celle d'un tiroir à cadavre ?). Vues successives rapides d'un gros billot métallique posé sur un plateau en métal, des instruments chirurgicaux : multiples ciseaux, pinces, 


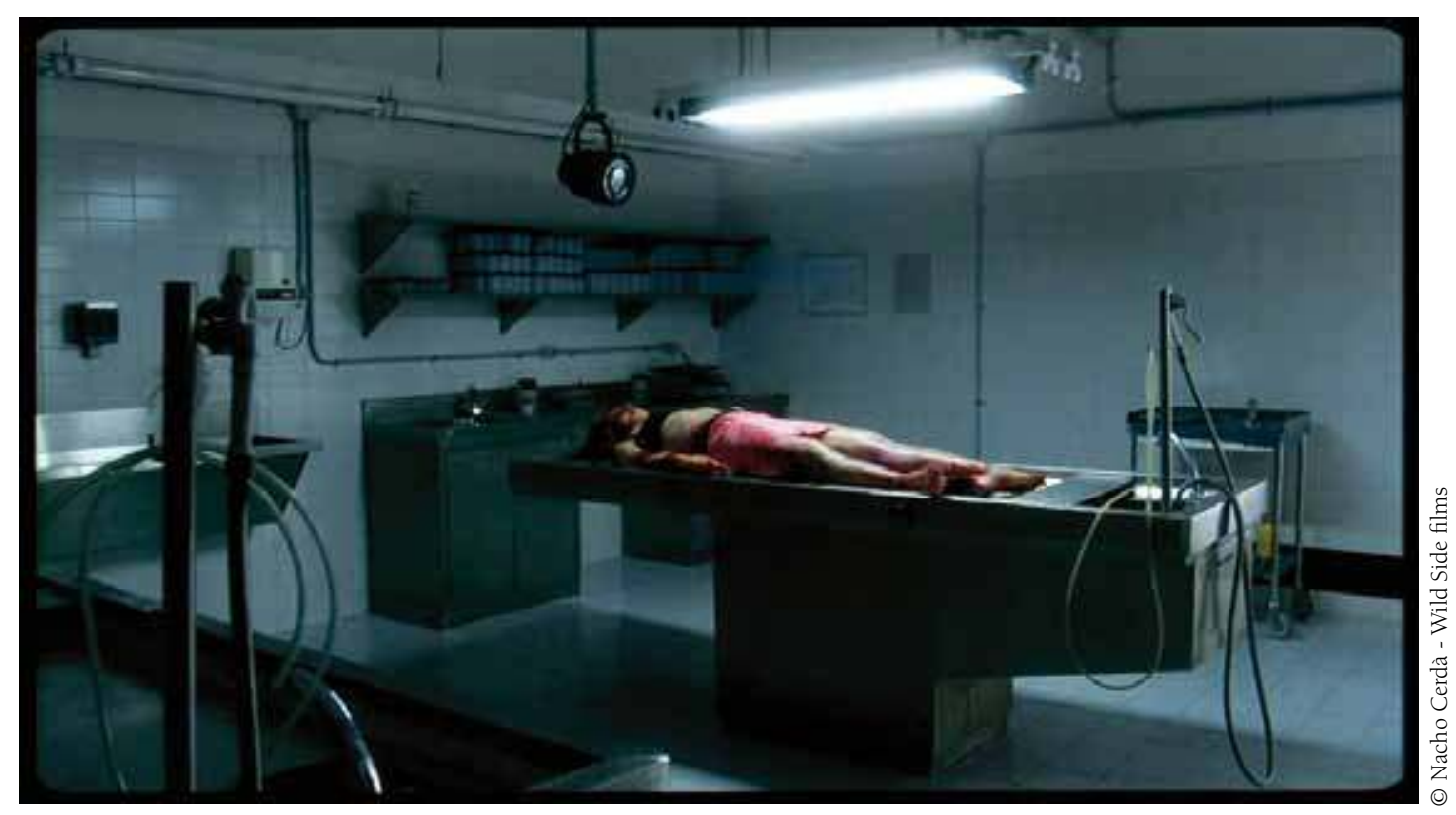

écarteurs, bistouris de différentes formes et tailles alignés les uns à côté des autres sur une surface métallique. Tout est ici encore parfaitement froid, ordonné et propre.

Le petit « ding » de l'ascenseur nous signale l'arrivée au niveau moins trois (est-il encore temps de revenir sur ses pas ?). Vue sur une étagère où sont disposés des bocaux en plastique transparent à bouchon noir de différentes tailles alignés sur une étagère et dans lesquels on aperçoit maintenant du liquide jaunâtre dans lequel trempent des morceaux d'organes. Se superpose en transparence une fiche formulaire, non encore remplie, concernant l'âge, le sexe, la taille, le poids corporel [du cadavre] et des différents organes en précisant à chaque fois le sexe (/) : cœur, poumons, foie, reins, rate, surrénales, testicules, ovaires, cerveau.

Niveau moins quatre. Vue sur des poignées et des robinets en métal rutilant : ici l'asepsie est réglementaire.

Niveau moins cinq. Un évier en inox, un autre en émail, chacun pourvu d'un robinet en inox ; un portant dans lequel sont placés des tubes de prélèvement en plastique avec des bouchons rouges. Bruit d'un enregistrement cardiaque avec graphiques en rouge de l'électrocardiogramme dont le mouvement commence par s'affoler pour se ralentir ensuite progressivement et finalement dessiner une ligne plate rouge tandis que le son devient une sonnerie stridente continue indiquant l'arrêt cardiaque définitif. Le Requiem de Mozart qui nous a accompagné jusque-là s'arrête, remplacé par ce son strident et rémanent. Nouveau claquement de porte. Une petite croix blanche sur fond noir s'inscrit et s'intègre aux mots du générique soulignés par une ligne rouge : produit, écrit et dirigé par Nacho Cerdà.

Niveau moins 6. Le générique est terminé, nous sommes arrivés dans les entrailles de l'hôpital, on ne peut descendre plus bas... Un jeune brancardier en tenue blanche d'hôpital avec son walkman sur la tête apparaît dans l'ouverture de l'ascenseur poussant devant lui son chariot brancard recouvert d'un drap d'où sortent les orteils du cadavre 
et l'étiquette du gros orteil. On entend ses pas et des bruits insolites. Il s'éloigne dans un couloir sombre. Un autre type de musique classique se fait entendre, moins solennelle que le Requiem. Le brancardier passe devant un couple, assis sur des chaises de salle d'attente, dont on subodore qu'il s'agit des parents de la jeune morte : la femme a blotti sa tête contre l'épaule de son compagnon et on l'imagine en train de pleurer silencieusement. La main d'un membre du personnel dont on ne voit pas le visage tend à l'homme qui s'en saisit en refermant la main sur elle, la croix suspendue à la chaîne. Seul élément personnel aperçu de la jeune fille, elle est rendue à ceux qui fondent son identité sociale, ses parents ; son corps, une fois qu'il a été parfaitement dépersonnalisé, appartient désormais au personnel de la morgue.

La croix, symbolise, on le sait, La Passion, ensemble des souffrances et supplices qui ont précédé et accompagné la mort du Christ en tant qu'être de chair à l'instar des simples mortels. Elle symbolise aussi l'âme rattachant les humains à la transcendance en fondant leur affiliation à la divinité. L'Église est l'institution qui se consacre à cette part intangible et immortelle des sujets et qui est ainsi susceptible de prodiguer assistance et consolation aux survivants, fonction présentifiée par la messe pour les morts qu'est le Requiem. Cette tâche la distingue fondamentalement de celle déléguée à l'institution laïque par excellence qu'est l'hôpital public, aspect souligné ici à la fois par l'interruption du Requiem et par l'absence d'échange de paroles entre les parents et l'agent hospitalier. Dans ce temple séculier, point de réconfort. Nous sommes confrontés à la froideur, la solitude et la désolation associées à l'anonymisation de la mort que les procédures hospitalières rigoureuses d'asepsie symbolisent si bien : la décontamination des corps est aussi bien physique que relationnelle. Si l'on estime si nécessaire d'ériger l'humanisation des hôpitaux en droit, c'est que la déshumanisation y règne en fait. Dans leur enceinte, les profanes, ici les parents, se distinguent immédiatement, par leurs vêtements, des professionnels portant les tenues si repérables du personnel hospitalier. Les « patients » se situent entre les mondes civil et professionnel et sont eux-mêmes répartis en deux cohortes, les vivants et les morts, ces derniers à la totale merci des professionnels.

\section{Une transgression légale}

De fait, nous voici ramenés dans la morgue où le jeune brancardier au walkman empaquette avec soin dans du plastique blanc le cadavre posé sur un plateau métallique sorti en partie du casier métallique de l'armoire à cadavres. Pas de sang ni sur le brancardier, ni sur le plastique. Son travail fait, il pousse le plateau dans le casier où le cadavre empaqueté et étiqueté est entreposé.

Nous sommes alors immédiatement mis en face d'une autre scène : un médecin légiste retire avec quelque difficulté une bague sur la main d'un cadavre masculin allongé encore habillé. On nous fait par la même occasion ressentir la rigidité cadavérique de ceux qui sont cantonnés à l'immobilité, l'horizontalité et la passivité, faisant contraste avec les « verticaux » à qui revient l'exclusivité du mouvement et de l'action. Les doigts du mort sont maculés de sang, un bracelet blanc d'identification enserre son poignet. La bague est déposée sur un petit plateau métallique au milieu des instruments de dissection. On entend les bruits métalliques des instruments et ceux des vêtements que l'on déchire. 


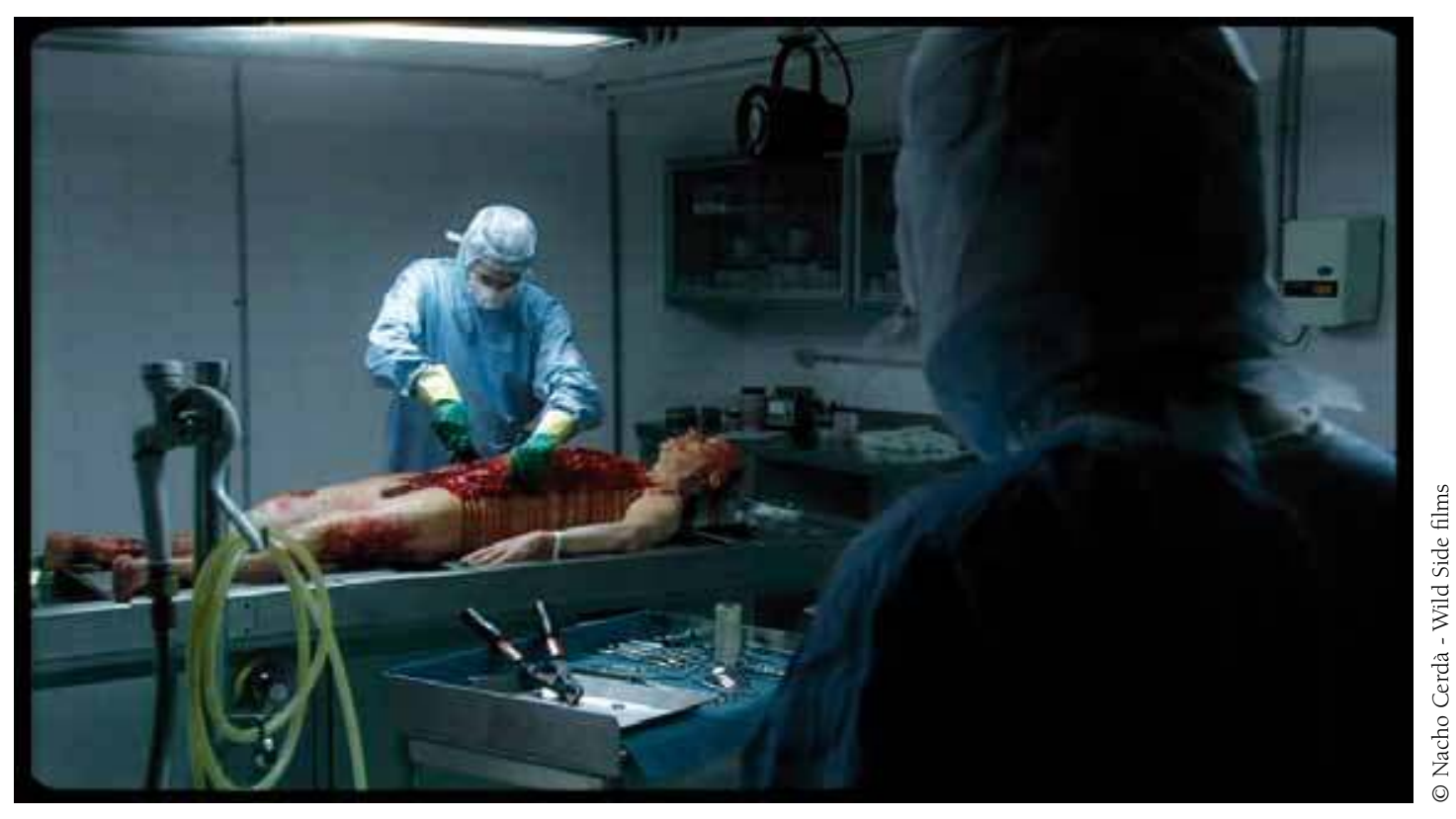

Les deux médecins légistes qui opèrent chacun sur leur cadavre respectif portent des blouses et des cagoules bleues, en papier tout comme leurs masques chirurgicaux dissimulant leur bouche et leur nez, et des gants verts bordés de jaune en caoutchouc semblables à ceux des ménagères. Ce sont des couleurs froides tout comme le blanc des murs et du sol carrelés et de l'émail de l'évier où les légistes se nettoient les mains, des bocaux en plastique, et le gris métallisé du matériel, instruments et mobiliers en acier ou en inox. Les couleurs chaudes, ce sont paradoxalement les cadavres dénudés qui les portent, ceux dont on voit les visages aux yeux ouverts et les sexes bien en vue : le rouge du sang recouvrant leur corps, les différentes nuances de marron de leur peau. Nous voyons et nous entendons les couteaux aiguisés par les légistes. Nous sommes confrontés régulièrement aux instruments, à la scie circulaire et à la ficelle utilisée pour rapiécer grossièrement les corps ouverts et dépecés, à l'instar de volailles que l'on recoud en cuisine après les avoir éventrés et farcis. Le corps est ici traité comme une viande à dépecer dont les légistes sont les bouchers. Chacun place la tête du cadavre dont il s'occupe sur un billot métallique afin de l'ouvrir à l'aide d'une scie circulaire dont nous entendons, nous qui sommes des spectateurs passifs à l'instar des sujets pourfendus, le bruit strident; nous voyons le sang gicler sur la blouse et le masque des légistes. Puis des pinces géantes sont utilisées pour écarter les os du crâne tandis que le bruit de décollement des tissus se fait entendre; le cerveau est retiré, disposé sur une balance métallique, et des chiffons sont enfoncés dans le crâne évidé générant des bruits de succion liés à l'absorption des liquides céphalorachidiens. Le crâne reste vide et béant. Les cages thoraciques et les abdomens sont ouverts au couteau et à la pince. La graisse jaune et les organes internes apparaissent.

Aux pieds des tables d'autopsie sont disposés des éviers en inox avec des tuyaux en caoutchouc destinés à nettoyer le sang au jet. Le travail répugnant va ici de pair avec une obsession pour la propreté soulignée par Cerdà dans ses commentaires. 
Paradoxalement ce souci de faire disparaître les traces du sale travail participe de la froideur, de la mise à distance, de la dépersonnalisation des individus traités en objets.

Le jeune brancardier au walkman, les mains dans les poches de son pantalon blanc, l'allure désinvolte jette un oil au travail des légistes, son visage exprime une légère inquiétude. Dans cet univers, il est d'ailleurs le seul être vivant dont nous voyons le visage dans son entier puisque nous ne percevons de celui des légistes, masqués et cagoulés, que les yeux. Il est à la fois l'intermédiaire entre les vivants et les morts, et le pont jeté entre les profanes et les vrais professionnels. Il observe les autopsies de loin, protégés par son walkman des bruits abominables provoqués par les gestes de profanation pratiqués sur les défunts qui sont, pour leur part, sourds, muets et aveugles. L'un des médecins légistes, que je nommerai $n^{\circ} 2$, regarde soudain drôlement le jeune homme qui s'éloigne, nous laissant seuls dans ce monde de la transgression légitime. Plus rien ne nous rattache maintenant à l'univers d'en haut. Nous voici coincés dans ce cloaque et nous devons abandonner toute espérance : nous ne pouvons que contempler ce que l'on nous impose de voir et d'entendre, et, à l'exemple de Dante, nous sommes devenus les yeux et les oreilles des morts suppliciés dans cet enfer.

L'un des légistes nettoie le cœur qu'il vient d'arracher et fait couler du liquide dans un récipient où il place ensuite l'organe. L'autre légiste se saisit du foie et des morceaux d'intestins qui traînent sur la table et les jettent dans le thorax ouvert, il y place aussi le cerveau et appuie sur le tout pour refermer les parois préalablement largement éversées $\mathrm{du}$ tronc. Lorsque les corps sont grossièrement cousus pour fermer leur thorax, les cadavres tressautent donnant l'insolite impression qu'ils s'animent et qu'ils sourient ${ }^{3}$. Le sang s'écoule dans l'évier et se mélange à l'eau du tuyau d'arrosage qui sert à nettoyer grossièrement les corps avant de les replacer dans leur housse en plastique fermée à l'aide d'un zip aux bruits si caractéristiques. Le travail légal du légiste consistant à mettre à nu les chairs et à les manipuler et, par là, à violer la sacralité des corps à des fins reconnues d'utilité publique, est terminé. Légiste $\mathrm{n}^{\circ} 1$, celui qui exerce son travail sans état d'âme mais honnêtement, incarnant ainsi la norme de la transgression légalement définie, disparaît du champ à l'instar du brancardier. Nous sommes donc prêts à descendre un peu plus profond dans l'horreur.

\section{La perversion}

Vue sur légiste ${ }^{\circ} 2$ malaxant dans sa main avec délectation des débris humains ensanglantés tout en regardant le pénis de «son » cadavre. Nous sommes introduits ici à une autre forme de transgression cette fois clairement prohibée par la loi : le plaisir éprouvé à manier les chairs pour satisfaire des pulsions personnelles indues. Les membres du personnel des morgues ont précisément peur, non seulement de banaliser leur expérience au point de ne plus ressentir ni crainte ni dégoût, mais pire encore de pouvoir être accusés d'en ressentir du plaisir (Laudanski \& Jeanjean, dans cet ouvrage). Ce que le réalisateur vise maintenant à nous montrer, c'est le passage au travers du miroir réfléchissant les questions obsédantes que se posent les profanes et que doivent habituellement combattre dans leur for intérieur les professionnels de la mort ${ }^{4}$ : quelles sont les motivations profondes des gens exerçant un tel travail ? Que se passerait-il si l'on franchissait la limite des gestes imposés et que l'on 
s'impose à soi-même, limite qui ne doit normalement pas être enfreinte sous peine d'être dans la perversion vis-à-vis des autres mais aussi, et plus fondamentalement encore, vis-à-vis de soi-même? En nous révélant l'autre côté du miroir, Cerdà va nous permettre d'entrevoir la tension éprouvée au quotidien par ces professionnels : ressentir l'effroi tout en le tenant à distance, tout faire pour préserver sa normalité en effectuant un autre travail dans le civil, tenter ainsi d'éviter la contamination possible de sa vie personnelle et affective par l'expérience vécue au contact de la putréfaction (Laudanski \& Jeanjean, ib.). Nous sommes également amenés à comprendre l'isolement dans lequel les confine la part abjecte de leur métier. Ils ne peuvent la partager qu'avec leurs semblables, les autres initiés.

La première partie de notre introduction dans la morgue visait à nous montrer de manière documentaire et aussi réaliste que possible le travail légal qui s'y déroule. Cerdà dit bien qu'il décrit alors ce qu'il a vu lors de son travail d'observation dans une morgue, sans inventer ni fictionnaliser, mais au contraire en retranchant les choses bien pires qu'il y a vues. C'est pourquoi, il a tourné dans une vraie morgue en utilisant tout le matériel qui s'y trouvait, seuls les cadavres sont faux et participent d'effets spéciaux tout à fait remarquables. Ce que Cerdà nous montre par la suite est d'un autre ordre : la fiction est introduite dans la morgue au moyen de la mise en scène soignée et particulièrement éprouvante d'actes pervers nécrophiles; elle réalise les fantasmes que les non professionnels de la mort peuvent aisément projeter quant aux transgressions potentielles qui peuvent y être accomplies. L'horreur fictionnelle rajoutée à l'horreur réaliste va paradoxalement réintroduire de l'affect, une émotionnalité qui avait préalablement été soigneusement évacuée en montrant des légistes accomplissant uniquement des gestes techniques sans échanger quoi que ce soit entre eux ou avec leurs cadavres. Dans la réalité, l'émotion s'exprime en fait au travers de plaisanteries incessantes, voire par des blagues que se font les agents du personnel. La plaisanterie, loin d'être l'expression d'une pure grossièreté, est une façon d'introduire du débordement contenu ; c'est un garde-fou permettant de réinjecter de l'humanité dans un univers voué à la déshumanisation. C'est précisément ce que Cerdà a souhaité éliminer de son scénario pour ne pas risquer de verser dans le grotesque; il ne voulait pas se faire complice des spectateurs en leur tendant gentiment la main par le biais du rire ou même de l'humour. Le grotesque aurait servi d'échappatoire, ce qui est le propre du cinéma gore ordinaire. C'est ce qu'a parfaitement compris l'acteur jouant le rôle de n², Pep Tosar, qui affirme que la scène du viol nécrophile est la plus difficile qu'il ait eu à jouer de toute sa vie. Il a dû aller puiser aux tréfonds de lui-même pour atteindre la vérité de son personnage, exigeant, avant et après la scène du viol nécrophile, de rester seul pendant de très longs moments, pour se préparer, puis pour récupérer. Cette vérité renvoie, d'après lui, à la solitude abyssale de cet homme et à son besoin corrélatif « d'absorber la force vitale de tout ce qui l'entoure, y compris celle des cadavres » :

«Si j’avais permis que mes mécanismes de défense m'éloignent de la réalité que je jouais, j’aurais frôlé le grotesque. On aurait fait une scène comique. La seule possibilité était de le faire pour de bon. C'était une ligne trop fragile pour le jouer avec moins de tension. ».

Soulignons aussi qu'au moment du tournage de cette scène, la plupart des membres de l'équipe, gênés ont quitté le plateau.

Raccord de champ blanc se fondant sur une vue du carrelage blanc qui recouvre les murs de la morgue. № 2 lit les fiches d'un dossier de ses yeux noirs aux sourcils épais éminemment inquiétants dans leur expression lourde. Il regarde en direction 
d'un des casiers où sont entreposés les cadavres et nous voyons celui portant l'étiquette « MartaArnau ». Il s'empresse de ramener ce nouveau cadavre encore engoncé dans sa housse de plastique blanc qu'il défait rapidement. Il dépose le corps sur la table d'autopsie et le regarde longuement. La morte est simplement vêtue d'un soutien-gorge noir et d'une jupe rose très courte pourvue d'une ceinture noire. Il caresse le visage maculé de sang aux yeux ouverts, s'arrête sur les lèvres, le menton. Il va fermer à clé une porte, prend dans son casier personnel une sacoche en cuir noir et retourne auprès de la morte. Il fait glisser l'un des ciseaux chirurgicaux le long de la ligne médiane de l'abdomen jusqu'au milieu du soutien-gorge qu'il coupe et retire. Il défait la ceinture, regarde la petite culotte blanche en haletant lourdement et la coupe. Le sexe de la jeune femme apparaît en plein milieu du champ. La musique du Requiem qui avait cessé lors de la fin du générique d'ouverture reprend sans couvrir pour autant le bruit des ciseaux et de la respiration du légiste. № 2 recommence à caresser le visage de la morte tout en saisissant un couteau. On ne voit plus que l'évier en inox mais on entend un bruit de décollement indiquant qu'il est en train de commencer l'autopsie avec son couteau. Le Requiem cesse laissant place aux seuls bruits de l'eau s'écoulant.

Plan noir indiquant un changement de champ. Nous voyons le carrelage blanc où des gouttes de sang tracent un chemin jusqu'à $n^{\circ} 2$ qui a posé sur la balance métallique le cerveau de la morte. $1 \mathrm{~kg} 283$. Il se dirige ensuite vers l'évier où il nettoie soigneusement son long couteau à grande eau. Il revient vers la table d'autopsie et caresse avec son tranchant la ligne médiane du visage de Marta alors qu'une musique de fond mécanique s'élève. Il passe la lame de son couteau sur le front, les lèvres, le menton, la ligne médiane du thorax, va vers les seins et fait le tour d'une des aréoles, puis descend et remonte à plusieurs reprises sur la ligne médiane de l'abdomen et du thorax dans un mouvement de va-et-vient répété de plus en plus rapide et enfonce finalement à toute force et à plusieurs reprises son couteau-godemiché dans le sexe de la jeune femme. Nous entendons dans

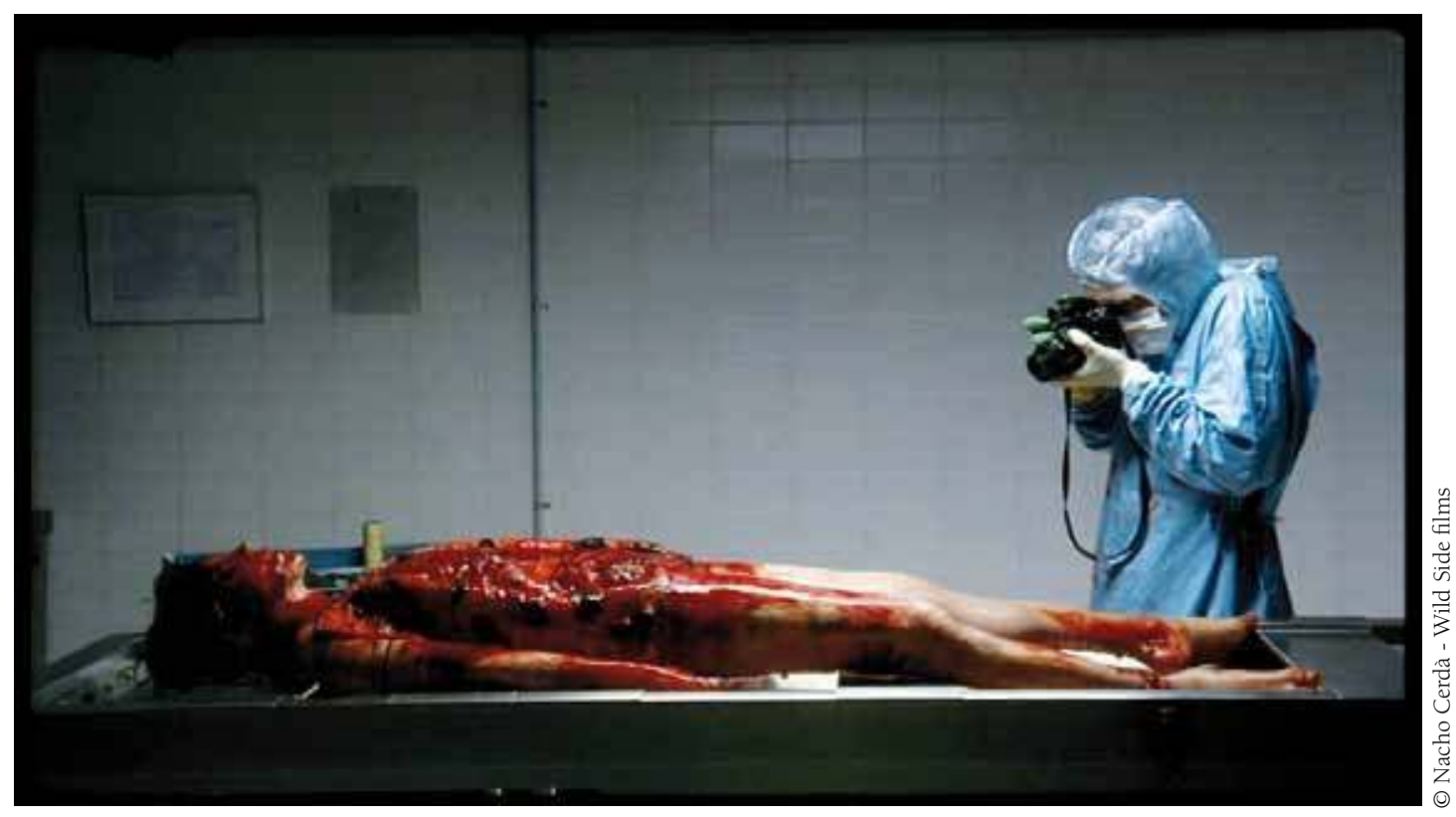


le même temps les bruits consécutifs de succion. Le sang coule dans l'évier. Le couteau finit par tomber au sol. Dans la scène suivante, nous découvrons qu'il a ouvert le thorax et l'abdomen transformés en marécage de chair humaine macérant dans le sang. Il triture le sang et ces chairs tandis que le Requiem reprend. Il glisse sa main droite sous sa blouse pour sortir son sexe (sans que nous ne voyions celui-ci) et se masturbe tout en malaxant de la main gauche les organes thoraciques de la morte tandis que nous entendons ses gémissements de plaisir. Il jouit et tombe à genoux alors que le Requiem se poursuit.

Champ sur le bac de l'évier en inox et sur la sacoche noire en cuir. Il prend des photos du cadavre complètement dénudé, ensanglanté et ouvert, puis du sexe de la morte. Le Requiem cesse. $\mathrm{N}^{\circ} 2$ dispose son Nikon au milieu de la table à instruments, revêt l'un de ses gants en caoutchouc, enduit ses deux doigts de la main droite de vaseline (pour un rapport anal ?), retire ses chaussures et son pantalon tout en conservant sa blouse et sa cagoule en papier bleu ainsi que son masque. Il se met au-dessus de la morte, lui fait l'amour tout en se prenant en photos en automatique (36 prises !). On n'entend les prises automatiques, et les halètements-gémissements de sa jouissance, le tout sans aucun support musical. Bruits et mouvements s'accélèrent, ses jambes sont nues mais il a conservé ses chaussettes. Cette scène est d'autant plus pénible qu'elle semble durer une éternité (près de deux minutes). $N^{\circ} 2$ finit allongé sur le cadavre comme s'il avait épuisé toute son énergie dans cette tâche.

Fondu noir. Le Requiem reprend. Au sol sont éparpillés le foie, une partie des intestins, de la graisse jaunâtre. Vue sur la boîte de pellicule photo vide puis sur le cerveau toujours disposé sur la balance. Vue sur la morte, les yeux et la bouche ouverts, complètement recouverte d'un voile de sang. Vue sur une bouteille de whisky ouverte posée sur le bord de l'évier. No 2 ramasse les débris humains tombés à terre et les dispose sur un petit plateau métallique. Il découvre le cœur et le met à part dans un petit sac en plastique transparent. Il remet les poumons, le foie, etc. dans le thorax de la morte, referme grossièrement le tout en rabattant la paroi éversée et remet la morte dans son sac en plastique blanc sur

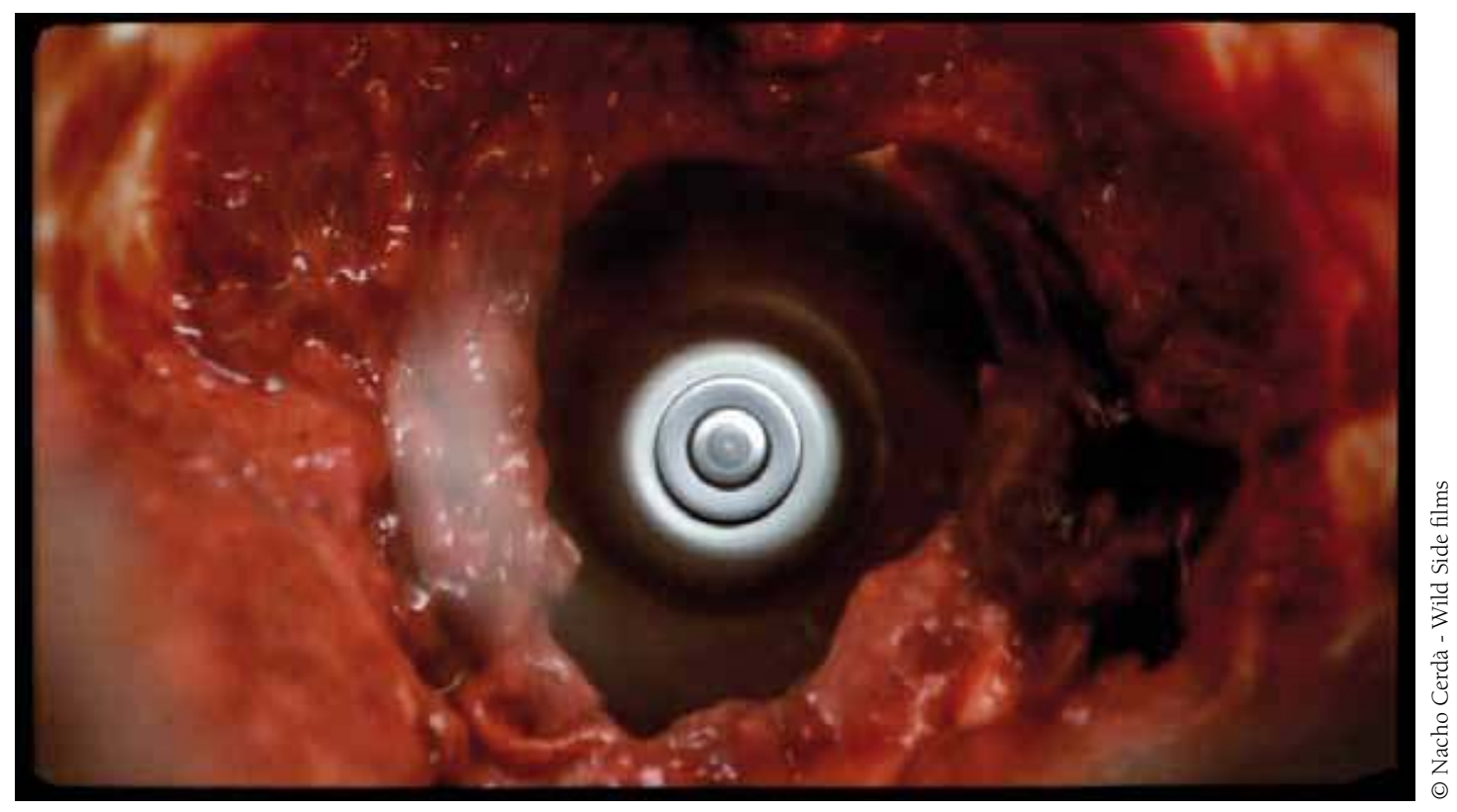


lequel est inscrit au crayon-feutre Arnau Marta. Puis il nettoie au jet d'eau les éviers et les tables métalliques. Longue vue sur la propreté retrouvée de la table d'autopsie, comme si rien ne s'était passé. Dépourvu de sa blouse en papier bleue, en vêtements civils, jean bleu et chemise noire, il jette à la poubelle ses gants en latex qu'il portait sous ses gants en caoutchouc, et se nettoie soigneusement les mains. Il glisse le petit sac contenant le cour dans sa sacoche noire en cuir. Vue sur l'évier en émail blanc dans lequel il s'est nettoyé les mains qui sont maintenant parfaitement propres et dont la robinetterie en inox est rutilante. Puis il sort de la morgue en éteignant les lumières. La musique du Requiem de Mozart s'arrête alors.

\section{Home, sweet home}

Plan noir. Bruit d'une moulinette électrique de cuisine puis vue occupant tout le champ sur la chair rouge en train d'être moulinée avec au centre « l'œil » en inox des couteaux de la moulinette en train de tourner nous regardant avec insistance comme l'œil de Dieu sur Caïn.

On entend $\mathrm{N}^{\circ} 2$ siffler gentiment son chien. Une porte s'ouvre sur un couloir blanc mais chaleureux aux murs décorés de gravures, on aperçoit dans une pièce attenante des étagères couvertes de boîtes d'alimentation et des bouteilles de vins. № 2 apparaît dans un pyjama d'intérieur blanc bordé de noir, très souriant et affable : c'est la première fois que nous pouvons le dévisager en entier. Un gros chien apparaît dans le couloir, haletant gentiment et se précipitant sur le plat en inox que son maître, tout en lui donnant quelques caresses, dépose sur le sol au-dessus de journaux. No 2 referme la porte du couloir nous laissant avec le chien qui mange bruyamment avec appétit. Pin-pon très assourdi d'une ambulance passant au loin. S'entrouvre alors la pièce où se trouve № 2 : un salon confortable. Les pieds posés sur sa table basse, il fume, regarde la télévision et manipule sa télécommande comme tout un chacun. On entend très faiblement le bruit d'un bébé pleurant quelque part tandis qu'apparaît un fondu noir et que nous entendons à nouveau de la musique classique, mais non le Requiem. Nous nous retrouvons dans le couloir déserté par le chien : son plat en inox contient quelques débris de viande crue, certains éparpillés sur les papiers journaux. Une vue rapprochée sur ces derniers révèle des notices nécrologiques maculées de débris de viande. L’une d'elles concerne Marta Arnau Marti ; surmontée d'une croix noire, elle précise que Marta est décédée le vingtcinq décembre et que ses parents, sa famille et ses amis adressent une prière pour elle. Le mouvement de la caméra nous fait entrevoir un gros titre beaucoup plus accrocheur annonçant qu'une jeune Indienne de 18 ans est devenue Miss Univers. Fondu noir puis la musique du Requiem reprend tandis que le générique de fin apparaît. 


\section{Le cœur a ses raisons que la raison ne connaît pas}

À première vue, tout se passe comme si le film surenchérissait dans la déshumanisation des cadavres. La première partie quasi documentaire, nous a transformés en témoins du traitement scandaleux et pourtant légal de la profanation qu'ils subissent. Rien ne semble ne nous avoir alors été épargné puisque le contraste entre, d'un côté, la froideur du décor et des instruments, l'absence totale d'expression émotionnelle des légistes et, de l'autre, le dépeçage méthodique et outrageant accompli redouble notre sentiment d'horreur. Et, pourtant, les gestes de profanation sacrilège perpétrés par le légiste nécrophile sont encore plus insoutenables que les précédents et rendus plus odieux encore du fait qu'il se complaît à les photographier et que les bruits de sa jouissance prolongée nous indisposent à l'extrême. Cependant, en suivant le devenir du cœur de la défunte, nous allons pouvoir constater que, par un effet paradoxal lié précisément au recouvrement de la transgression légale mais impersonnelle par une transgression parfaitement illégitime mais hautement personnelle, les chairs de la défunte vont être réhumanisées 5 .

Le cour et ses battements sont symboliquement associés, dans notre culture, au ressenti émotionnel, à l'attachement et à l'amour, toutes choses qui semblent totalement bannies du film où, en apparence, c'est le cœur en tant que pur organe anatomique qui prend le pas sur l'expression des sentiments. Pourtant il se fait entendre dès les premiers instants, au cours même du générique présentant avec minutie le sujet du film. C'est d'ailleurs l'arrêt de ses battements, auxquels se substitue la sonnerie électronique de l'électrocardiographe, qui signe l'arrêt de mort et l'entrée dans la morgue. Et c'est après avoir fait l'amour, certes d'une façon des plus violentes, à la jeune morte que le légiste pervers découvre le cour de la défunte et le saisit. Or, dans ce deuxième volet, nous sommes dans la fiction, ce qui nous autorise à déchiffrer l'imaginaire sous-jacent au symbole utilisé : alors qu'il se débarrasse hâtivement des autres restes humains, il prend tout particulièrement soin de ce morceau de choix qu'il glisse dans sa sacoche après l'avoir mis dans un petit sac transparent. Il le ramène dans son petit nid douillet et c'est avec affection qu'il en fait offrande à son chien bien aimé après l'avoir passé dans son mixeur personnel. Son appartement, bien qu'il soit propre, n’a rien de l'aspect aseptisé de la morgue. $N^{\circ} 2$ qui a définitivement quitté sa tenue professionnelle pour réintégrer le civil (et la civilisation ?) et endosser un confortable pyjama n'est plus obsédé par la propreté : il ne s'empresse pas de nettoyer les débris humains restés sur les papiers journaux et dans la gamelle. Chez lui, la saleté relative des surfaces ne paraît pas l'incommoder. Le personnage maintenant affable a recouvré son visage et des sentiments humains ordinaires : nous le voyons enfin sourire et nous l'entendons siffloter. Il a réintégré un univers où les sons sont ordinaires et humains : pin-pon lointain d'une ambulance, pleurs d'un bébé, bruits de télévision sont autant d'indice que nous avons rejoint la surface terrestre où s'égayent les vivants. Et c'est dans cette atmosphère familière et intime qu'il a rapporté l'organe des sentiments de Marta qui avait été préalablement reléguée dans un tiroir interchangeable de l'armoire frigorifique à cadavres.

Dans ce final, le chien si vivant et sympathique ${ }^{6}$ ressuscite en quelque sorte celui qui a été tué par la défunte lors de l'accident de voiture inaugural qui leur a été fatal à tous deux. En ingérant la chair de la jeune femme, l'animal domestique bien aimé lui permet d'être incorporée et, ainsi, réintégrée au cycle de la vie : la résurrection de sa chair est assurée. Je me sens d'autant plus justifiée à filer la métaphore de la résurrection par 


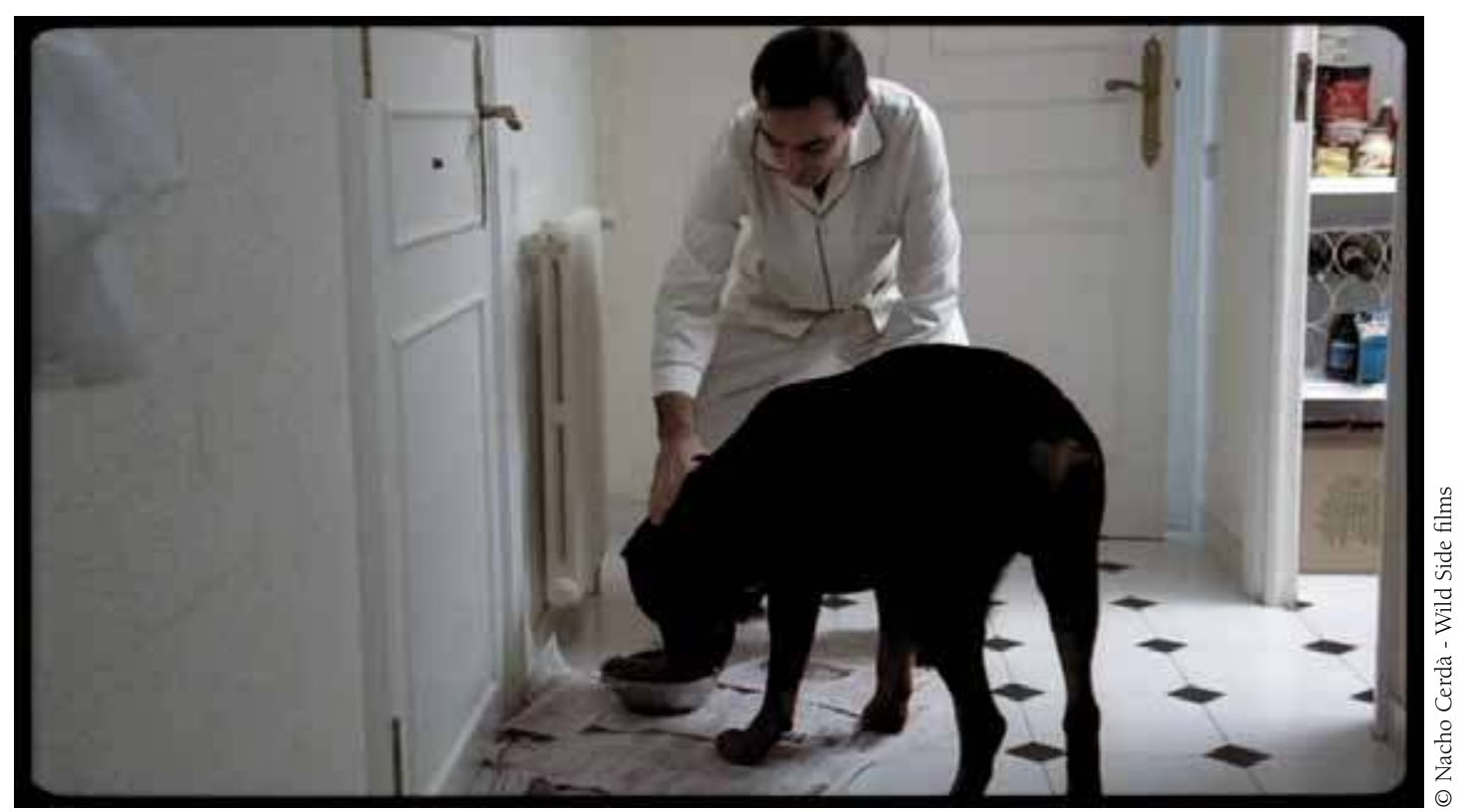

ingestion qu'avant de faire ce film, Cerdà avait eu l'intention d'en faire un autre associant le sexe au cannibalisme. Par ailleurs, il repère lui-même l'étrange relation existant dans Aftermath entre sexe, mort et obsession de rendre toute chose immortelle : en se prenant en photo au cours de son rituel macabre, le légiste s'immortalise dans les bras de la morte.

Quoi qu'il en soit, c'est en retrouvant la chaleur du foyer ayant accueilli son cœur que Marta recouvre sa qualité de sujet humain possédant une identité personnelle et relationnelle : la notice nécrologique qui lui est dédiée exprime la prière que lui adressent publiquement ses parents, sa famille et ses amis, elle qui est morte le jour de la naissance du Christ, et la croix surmontant cette annonce se substitue à celle dont elle a été dépossédée à la morgue. Son corps périssable est certes voué à la dissolution mais son âme est sauve : élue d'entre tous les morts de la morgue, suppliciée plus que les autres, elle est assurée d'entrer sans délai au paradis.

Il est donc bien question ici d'une forme de consolation et de résurrection, des éléments religieux bel et bien évoqués au travers du Requiem de Mozart qui ponctue de façon significative la succession des scènes. En effet, quand Dieu invoqué par le chœur s'absente, le monde n'est plus que bruits. De fait, l'accompagnement sonore est un autre repère essentiel pour déchiffrer les allers-retours entre déshumanisation et réhumanisation. Notons tout d'abord que la musique religieuse du Requiem confère une solennité à l'événement que représente le passage de la vie à trépas au cours des génériques d'ouverture et de clôture du film. Entre ces deux moments, elle sert à souligner, lorsqu'elle cesse de retentir, d'une part l'abomination des bruits accompagnants les gestes profanateurs de transgression légale et perverse perpétrés dans la morgue, d'autre part, la trivialité des sons qui émaillent l'univers familier de $\mathrm{n}^{\circ} 2$. Le choix de cette musique est particulièrement adéquat, non seulement parce qu'elle est une messe pour les morts mais également parce qu'elle évoque la mort prématurée, en décembre, comme celle de Marta, de son auteur décédé lors de sa composition. 


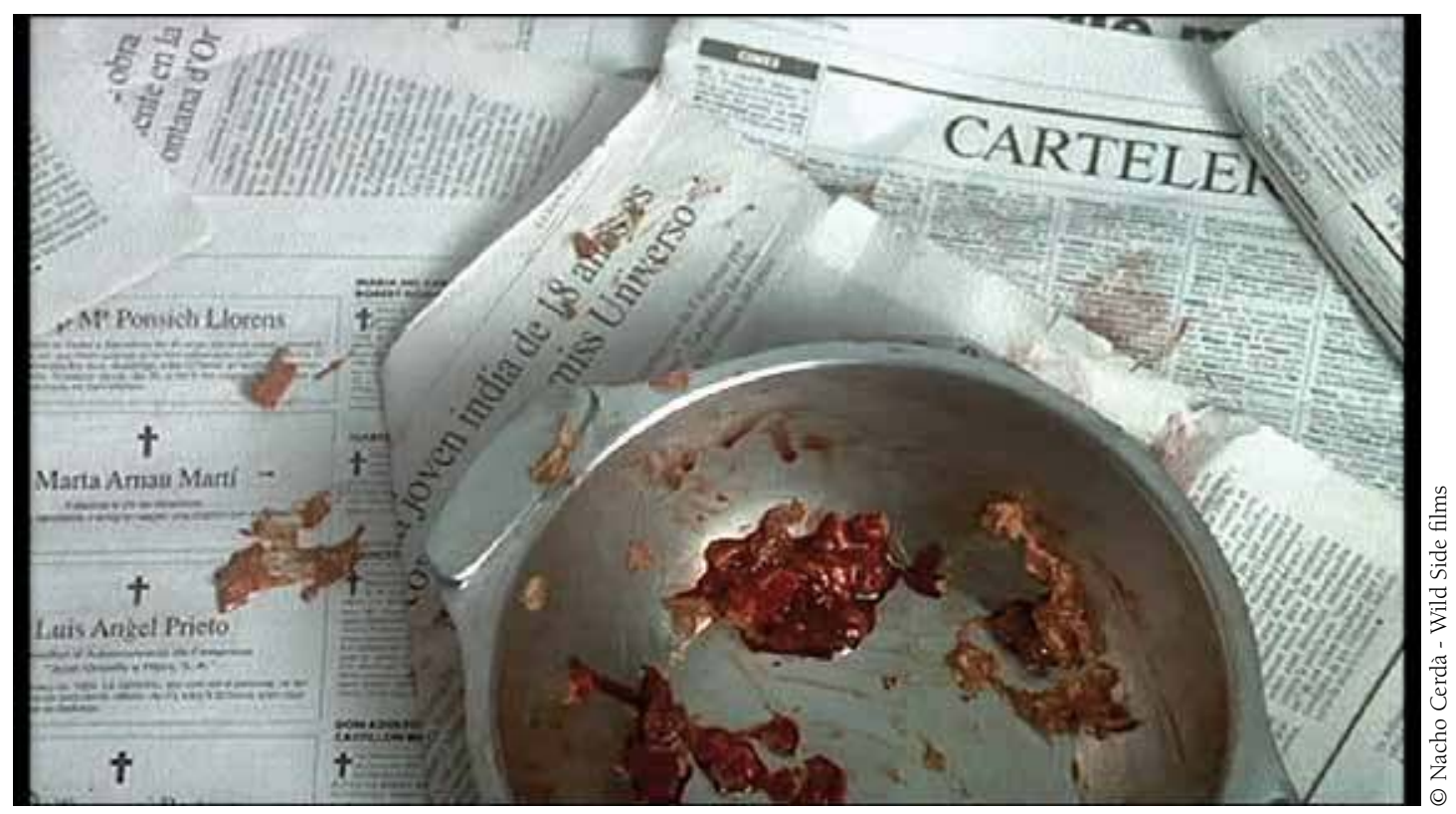

Nous voyons donc que le redoublement de la transgression par les actes illicites commis par un individu pervers apparaît comme un moyen fictionnel paradoxal pour réhumaniser ce qui a été déshumanisé par le traitement anonymisant médico-légal. L’horreur surajoutée à l'horreur a suscité, en outre, chez nous des émotions intenses compensant leur absence apparente au sein de l'institution hospitalière. C'est ce qu'exprime à sa manière un des fans de ce film culte :

« Aftermath is not enjoyable in any way. And that is why I liked it so much. [...] I've seen a lot of depraved stuff as the insatiable schizoid cinemaphile that I am. But Aftermath remains the ONLY film I can remember that has EVER kept me so enraptured and revolted at the same time. I've never seen a movie before [...] that has made me lose my appetite. Aftermath did just that. [...] The gore in Aftermath is [...] realistic to the point of being highly unsettling. [...] It is all depicted in a very hard, cold, hollow way. [...] The first time you watch it you sit there in silence, enveloped by what you're seeing. [...] Aftermath is a bleak, unnerving, disturbing film. It is not meant to entertain you. It is meant to move you. It is meant to make you feel that sick feeling in the pit of your stomach. »

Nous voyons donc que le redoublement de la transgression légale mais impersonnelle et, par là, anonymisante, par une transgression illégitime mais personnalisée (la perversion) constitue le dispositif d'humanisation du cadavre auquel contribuent les films d'horreur, un dispositif remédiant de manière efficace à la distanciation aseptisante du traitement médicolégal de la mort. Il revient donc à ce genre de films le mérite de restituer la dimension oblitérée de la mort, l'effroyable putréfaction à laquelle renvoie le cadavre maintenue à distance du monde profane par le processus de civilisation des mœurs (Elias 1973 [1969]) et, par la même occasion, de nous affecter profondément sans verser, dans ce cas précis, dans le pathos ou le grotesque. Demeure le pathétique susceptible de nous préparer à supporter la confrontation avec le cadavre de nos proches à laquelle il est peu probable que nous puissions échapper. 


\section{La chair et le sacré}

Les ethnologues rangent les rites dans le registre du religieux, et tout particulièrement ceux qui mettent en jeu le corps. De ce point de vue, les institutions médicales participent d'une forme certaine de religion laïque dans les sociétés occidentales contemporaines (Moisseeff 2013). Les travaux pratiques d'anatomie et de dissection des cadavres constituent la première étape de la trajectoire initiatique suivie par les officiants du culte occupant le plus haut grade, les médecins (Godeau 2007) : ils les confrontent d'emblée et de façon très crue à la mort et, par là, à la manipulation impudique des corps qu'ils n'auront de cesse de pratiquer sur les vivants et les morts. Cet apprentissage se poursuit, pour ceux ayant réussi le concours de l'internat, dans les salles de garde où le folklore pornographique et scatologique des carabins est de règle. Les rites ouvrant et fermant le temps de l'internat, bien que fondés sur la débauche, sont appelés baptême et enterrement.

Cette référence insistante au sacré, associée à des pratiques éminemment transgressives, n'est de mon point de vue aucunement fortuite si l'on se réfère à la conception du sacré de Bataille (1957, 2004). Dans cette perspective, le sacré est nécessairement rattaché à la transgression, c'est-à-dire qu'il renvoie au franchissement d'une frontière entre ce qui peut être exhibé ou pratiqué et ce qui doit ordinairement être tenu secret, maintenu à distance des sens et, notamment, de la vue et du toucher. Lorsque cette frontière est violée, on est dans l'extraordinaire qui peut être organisé comme tel au moyen de conventions socialement reconnues, comme c'est le cas au cours de l'examen médical ou des interventions chirurgicales ou médico-légales. L'exhibition de l'intimité corporelle est, de fait, celle qui est la plus susceptible de renvoyer à une transgression. C'est pourquoi, il y a une contiguité entre le sacré et, d'une part, l'érotisme, d'autre part, la mort, les trois pouvant se trouver conjugués dans des circonstances extrêmes, ce à quoi renvoie l'imagerie des œuvres de Sade, Bataille et Guyotat mais aussi du film dont il est ici question. Selon moi, ce qui lie ces phénomènes est la présence excessive du corps en tant que tel, c'est-à-dire sans médiation.

Ainsi le poids de la corporéité en excès du cadavre révèle une intimité que la présence de la subjectivité du sujet vivant qui l'animait masquait avant sa mort. Dans cette perspective, le sacré renvoie à l'exhibition de l'intime au cours de laquelle la présence lourde et concrète du corps au travers de sa nudité et/ou de ses sécrétions telles que sang, sperme, urine, excréments est essentielle, par opposition aux représentations distancées du corps et de ses substances que nous offre la liturgie chrétienne. Les salles d'urgence, les blocs opératoires, les morgues et les salles d'autopsie renverraient donc beaucoup plus qu'on ne le croit, et en dépit de toute la sophistication technologique de l'outillage utilisé, à des lieux où le sacré est à l'œuvre.

L'exhibition de l'intime confronte ceux qui en sont témoins à une altérité radicale qui met d'autant plus mal à l'aise qu'elle fascine et dégoûte à la fois, toutes choses qui ont précisément à voir avec la conscience d'enfreindre l'interdit marquant la frontière qui sépare ce qu'on a habituellement le droit de percevoir ou de faire et ce qui ne peut l'être qu'en des circonstances exceptionnelles. La conscience de transgresser ce qui est d'ordinaire un interdit joue un rôle essentiel dans toutes les procédures ritualisées mises en place dès qu'il est question d'opérer sur les corps. Pour qu'il y ait transgression par rapport aux codes ordinaires de sociabilité, il faut qu'une limite soit franchie et, pour ce faire, il est nécessaire qu'elle soit, dans le même temps, maintenue. C'est cette limite 
que le personnel médical est habilité à franchir grâce à l'initiation qui lui en ouvre le droit et dont on comprendra alors que le sexe et la mort y jouent un rôle fondamental (Godeau ibidem).

La froideur affective résultant des mesures aseptisantes mises en place dans les institutions médicales et médico-légales, si bien mise en évidence par Cerdà, est d'autant plus choquante que la matière qui en fait l'objet est humaine. Le scandale procède de la transformation d'un sujet en chose, en morceau de viande. Ces mesures sont le corollaire nécessaire à la distance requise d'un personnel constitué de non intimes à qui l'on délègue la tâche sacrilège de faire intrusion dans les intimités de ceux qui leur sont étrangers. Des gestes affectueux en cette occasion cantonnent aisément à la grossièreté et à la goujaterie, d'où l'interdiction d'avoir des relations sexuelles avec ses patients. C'est donc en vertu de la transgression légale à laquelle ils sont autorisés qu'ils se doivent de maintenir une distance affective. Cette barrière protectrice est la condition même permettant aux praticiens de continuer à exercer leur art transgressif sans faillir. Mais du point de vue d'observateurs extérieurs, cette mise à distance, protectrice tant pour les patients que pour les praticiens, est aisément perçue comme de la froideur gratuite.

Une façon de s'en sortir est de pratiquer un autre type de transgression, celle de l'humour, qui se surajoute à la transgression médicale. Il peut aisément être perçu, là encore, comme pure grossièreté. Pourtant, il sert fréquemment à réhumaniser la relation soignant-soigné, en exprimant l'humanité du praticien face à l'humanité de son patient. L'humour le plus grossier exprimé en groupe et hors champ est également un garde-fou lorsqu'il est exercé à distance par des praticiens ayant à vivre des scènes extrêmes les confrontant à l'urgence, à la crainte de perdre la bataille contre la mort, et à des tableaux particulièrement insoutenables. C'est ce qu'exprime la chanson de carabin que j'ai mise en exergue. S'y pencher est beaucoup plus intéressant et instructif qu'il n'y parait au premier abord. Notons tout d'abord que la puanteur évoquée est bien gentille : les salles d'autopsie seraient des paradis si elle ne concernait que les pieds... Par ailleurs, la parole est d'abord donnée au macchabée : à lui l'honneur de gueuler qu'il s'emmerde ici. Et si on le menace de le disséquer avec un spéculum, c'est qu'il est bien question de rappeler la réticence naturelle à aller voir dans ses entrailles ce qui est censé y rester. Le spéculum sert à regarder à l'intérieur du sexe de la femme, à jeter un œil sur cette origine du monde si énigmatique d'où jaillit la vie alors même qu'il est question de l'intimité d'un cadavre euphémisé. Et le pâté dans lequel on va le transformer est cette bouillie à laquelle aboutie la dissection requise par l'initiation de ces novices, si peu ragoûtante pour eux qu'elle les fera « dégueuler »... Ce qui s'exprime dans cette chanson aux tonalités joyeuses et rigolotes - et il est vrai qu'il est très plaisant de l'entonner en chœur -, ce sont donc les dessous de la basse besogne dont le commun des mortels est si bien préservé de nos jours en Occident. Si les gardiens du secret en tire gloire et privilège, c'est qu'elle sous-tend, malgré tout, la sacralité de leur fonction, une chose que nous a permis de rappeler une fiction bien mauvais genre. 


\section{NOTES}

Photo d'ouverture: Chanson d'étudiants Illustration extraite de l'ouvrage Chanson d'étudiants, auteur inconnu : 247-250

Toutes les photos sont extraites du DVD français La trilogie de la mort : Déclinaisons morbides. Courts métrage de Nacho Cerdà, production Wild Side films, Espagne.

1. Voir à ce sujet les auteurs auxquels font référence Déchaux 2001 et Memmi 2011.

2. Sur la façon de représenter la localisation spécifique de la morgue - hors du temps et de l'espace des simples mortels - dans les fictions horrifiques, voir Astic 2011.

3. Cette « animation » des cadavres et leurs couleurs «chaudes » renvoient à l'intentionnalité de Cerdà : "I wanted the dead to look alive and the alive to look dead. ». Toutes les références aux propos, intentions et ressentis de Cerdà, des acteurs et de l'équipe de tournage sont tirés des bonus du DVD du film dont on pourra retrouver un court extrait sur le site internet : http://www.horschamp.qc.ca/offscreen/nacho.html

4. Les ouvrages de Ganche 2012 [1909] et Hennig 2007 [1979] sont à cet égard particulièrement riches d'enseignements.

5. Là encore, les propos de Cerdà viennent appuyer cette perspective : « (...) it's a film against violence. It's a film against the manipulation of the human body, that's it. »

6. En dépit du fait que ce chien soit un rottweiler, une race considérée comme des plus dangereuse, comme l'a souligné un lecteur anonyme, Cerdà précise dans les commentaires de son film qu'il s'agit du chien d'un ami. En effet, à l'opposé du tournage de la première partie du film qui s'est déroulé dans une vraie morgue, les scènes de la seconde partie ont été tournées dans la maison de vacances des parents du réalisateur, au bord de la mer, là où, enfant, il passait tous ses étés; il y a réuni ses parents et les membres de son équipe. Les pyjamas portés par l'acteur sont les siens et, comme il le dit lui-même : "C'était une vraie réunion de famille! » Il s'agissait donc bien ici de créer une atmosphère familiale apaisant et joyeuse en parfait contraste avec la première partie si éprouvante. 


\section{POUR CITER CET ARTILLE:}

Moisseeff, M. 2013 Requiem pour une morte : Aftermath (Cerdà 1994). Ou l'art paradoxal de réhumaniser le cadavre, in H. Guy, A. Jeanjean \& A. Richier Le Cadavre en procès, TechniquesE Culture 60 : 160 -179.

\section{RÉFÉRENCES}

Astic, G. 2011 La Mort sans dessus dessous. La morgue dans l'horror contemporaine. In J. Goffette \& Guillaud L. L’imaginaire médical dans le fantastique et la science-fiction. Paris : Bragelonne : 363-376.

Bataille, G. 2004 Romans et récits. Paris : Gallimard.

— 1957 L'Érotisme. Paris : Minuit.

Déchaux, J.-H. 2001 La Mort dans les sociétés modernes : la thèse de Norbert Elias à l'épreuve, Ethnologie française 51 (1) : 161 à 183.

Elias, N. 1973 [1969] La Civilisation des mœurs. Paris : Calmann-Lévy.

Ganche, E. 2012 [1909] Le Livre de la Mort à l'hôpital -à l'amphithéâtre -à la morgue - au cimetière. Aiglepierre : La clef d'argent.

Godeau, E. 2007 L'Esprit de corps. Sexe et mort dans la formation des internes en médecine. Paris : Maison des sciences de l'homme.

Hennig, J.-L. 2007 [1979] Morgue. Enquête sur le cadavre et ses usages. Paris : Verticales : 30-31.

Memmi, D. 2011 La Seconde vie des bébés morts. Paris : Éditions de l'EHESS.

Moisseeff, M. 2013 La Chair, le sacré et le culte de l'homme dans les sociétés occidentales contemporaines, Revue du MAUSS semestrielle 41 : 173 à 192.

\section{RÉSUMÉ}

Requiem pour une morte : Aftermath (Cerdà 1994) ou l'art paradoxal de réhumaniser le cadavre. La mort, loin d'être devenue un sujet tabou dans les sociétés occidentales contemporaines, comme il est d'usage de l'affirmer depuis quelques décennies, est au contraire rendue omniprésente dans les médias : elle fait la une des journaux papier ou télévisées, constitue la trame de nombreuses fictions littéraires et cinématographiques, de séries télévisées américaines très prisées et de témoignages autobiographiques, tandis que tout un chacun est sommé, de manière itérative et insistante, de faire un travail de deuil et/ou de mémoire vis-à-vis de ses chers défunts. En revanche, il est bien vrai que la réalité concrète du cadavre tend, elle, à être occultée, phénomène qui ne peut échapper à l'ethnologue travaillant dans des sociétés autres où sa présence préside à la structuration des rites funéraires et à la gestion des réactions émotionnelles des proches. Dans l'Occident d'aujourd'hui, le cadavre humain et son traitement tendent, en effet, à être l'exclusive des institutions médicochirurgicales et médicolégales, un fait, par contraste, fort peu relevé. Les réactions émotionnelles provoquées par la perte et la mort tendent, pour leur part, à être gérées à distance de cette présence lourde et abjecte de la décomposition de l'être aimé, notamment au travers des conseils prodigués par les psy en tous genres. Cet article propose de voir dans la multiplication des films d'horreur une façon de reconnecter l'abjection du corps mort avec les réactions fortes qu'elle suscite lorsque les 
personnes y sont directement confrontées. Or, s'il revient aux institutions médicochirurgicales de faire le "sale boulot" à l'écart du monde profane pour rendre aux morts un aspect tolérable, il revient, par contre, aux films d'horreur de réintroduire la dimension effroyable des corps morts en voie de putréfaction. C'est du moins l'hypothèse que tentera de soutenir cet article en montrant que si la gestion du cadavre renvoie, dans le monde réel, à des formes de transgression légitimées par l'appareil juridique, il faut ajouter de la transgression à la transgression légitime pour restituer, sur un plan fictionnel, son véritable aspect horrifique et son aptitude à nous affecter profondément. Et quoi de plus transgressif, de ce point de vue, qu'un viol perpétré par un médecin légiste nécrophile dans une morgue, sujet même du court métrage du réalisateur espagnol Nacho Cerdà analysé ici?

\section{ABSTRACT}

Requiem for a Dead Lady: Aftermath (Cerdà 1994) or the Paradoxical Art of Rehumanising the Corpse. Death, far from being a taboo topic in contemporary Western societies, as has been commonly asserted over the past few decades, is on the contrary omnipresent in the media. Death daily makes the headlines in newspapers and on television, and provides the scaffolding for popular American TV series and autobiographical testimonials, while every one of us is repeatedly and insistently summoned upon to work out our grief and the memories we have of our dearly departed. On the other hand, what is true is that the concrete reality of the corpse tends to be obscured and concealed. This is particularly obvious to the anthropologist familiar with other societies in which the dead body's presence presides over the organisation of funerary rituals and the managing of close ones' emotional reactions. Indeed, in the present-day West, the human corpse and the treatments it undergoes tend to be handled by medical and forensic institutions exclusively, something which, by contrast, is rarely acknowledged. Emotional responses to death and loss tend to be managed at a distance from the weighty and wretched presence of decomposing loved ones, notably with the help of psychotherapists of various stripes. This article proposes to envisage the marked expansion of horror films as a way of reconnecting the abject character of the dead body with the strong reactions it gives rise to when people are directly confronted with it. Whereas it falls to medical-related institutions to do the "dirty work" at a remove from the profane world in a way that makes the dead more tolerable, horror films act to reintroduce the terrifying qualities of putrefying lifeless bodies. This article will present evidence in support of this hypothesis by arguing that whereas the handling of the corpse in the real world entails forms of transgression legitimized by the legal apparatus, it is necessary to add a further transgression to this legitimate transgression in order to reintroduce, in the fictional realm, the dead body's truly horrific nature and its ability to deeply affect us. And what could be more transgressive, from this point of view, than a rape carried out by a necrophilic medical examiner in a morgue, the very subject of the short film by the Spanish director Nacho Cerdà which this text proposes to analyse?

\section{MOTS CLÉS}

cadavre, mort, deuil, fiction, transgression, nécrophilie, morgue, autopsie, films d'horreur, institutions médicolégales

\section{KEYWORDS}

corpse, death, mourning, fiction, transgression, necrophilia,morgue, autopsied, horror movies, medical and forensic institutions 